\title{
The interplay between the charged Higgs and squark-gluino events at the LHC
}

\author{
Nabanita Bhattacharyya ${ }^{(a) 1}$, Amitava Datta $\left({ }^{(a) 2}\right.$, Monoranjan Guchait $^{(b) 3}$, Manas Maity ${ }^{(c) 4}$ \\ and Sujoy Poddar ${ }^{(d) 5}$ \\ (a) Indian Institute of Science Education and Research, Kolkata, \\ Mohanpur Campus, PO: BCKV Campus Main Office, \\ Mohanpur - 741252, India. \\ (b) Department of High Energy Physics, Tata Institute of Fundamental Research, \\ Homi Bhabha Road, Mumbai - 400005, India. \\ (c) Department of Physics, Visva-Bharati, \\ Santiniketan - 731235, India. \\ (d) Netaji Nagar Day College, \\ 170/436, N.S.C. Bose Road, Kolkata - 700092, India.
}

\footnotetext{
${ }^{1}$ nabanita@iiserkol.ac.in

2adatta@iiserkol.ac.in

3 guchait@tifr.res.in

${ }^{4}$ manas.maity@cern.ch

${ }^{5}$ sujoy_phy@iiserkol.ac.in
} 


\begin{abstract}
In some extensions of the standard model with extended Higgs sectors, events from new particle production may pass the selection criteria for Higgs search in different channels at the LHC - $14 \mathrm{TeV}$ and mimic Higgs signals. This intriguing possibility is illustrated by PYTHIA based simulations using several representative points in the parameter space of the minimal supersymmetric standard model (MSSM) including a point in the minimal supergravity model consistent with the Dark matter (DM) relic density data. Our simulations explore the interplay between the charged Higgs signal and typical squark-gluino events. We argue that the standard selections like the one based on the polarization properties of the $\tau$ 's from charged Higgs decay, though adequate for handling the SM background, may not be very efficient in the presence of SUSY backgrounds. We then propose an alternative search strategy based on pure kinematics which sufficiently controls both the SM and the MSSM backgrounds. For charged Higgs masses $\left(H^{ \pm}\right)$in the deep decoupling regime $\left(600 \mathrm{GeV} \lesssim m_{H^{ \pm}} \lesssim 800 \mathrm{GeV}\right)$ this method works well and extends the LHC reach close to $800 \mathrm{GeV}$ for an integrated luminosity of $30 \mathrm{fb}^{-1}$ with or without the SUSY background. For a lighter charged Higgs a judicious combination of the old selections and some of the cuts proposed by us may disentangle the Higgs signal from the squark-gluino backgrounds quite effectively.

PACS no:12.60.Jv, 12.60.Fr, 14.80.Cp.
\end{abstract}

\title{
1 Introduction
}

The Standard Model (SM) has a single neutral Higgs scalar in the spectrum. In contrast many extension of the SM have multiple Higgs bosons, both charged and neutral. Thus the discovery of at least two neutral Higgs bosons and / or a single charged scalar would unambiguously indicate physics Beyond Standard Model (BSM). Search for these particles playing pivotal roles in electroweak symmetry breaking is, therefore, a high priority programme at the Large Hadron Collider (LHC) running at $14 \mathrm{TeV}$.

Almost all the models with an extended Higgs sector have particles other than the quarks and leptons in the SM. They are expected to be produced at the LHC. The question that 
naturally arises is whether new particle production would create some final states which may pass the selection criteria designed for Higgs search for taming down the SM background only. The "Higgs signal" at the end of the day then would be an admixture of genuine Higgs events and the "new physics" backgrounds. It is therefore important to improve the search strategy which eliminates the SM as well as new backgrounds without paying too much price for the Higgs signal.

A case in point is models with supersymmetry (SUSY) [1]- one of the most well motivated extension of the SM. The Minimal Supersymmetric Standard Model (MSSM) has two neutral scalars $(h$ and $H)$, one neutral pseudo scalar $(A)$ and two charged scalars $\left(H^{ \pm}\right)$in the Higgs sector $[1,2]$. The prospective signals from these bosons at the LHC have been studied in great details $[2,3,4]$. It needs to be emphasized that in addition to these bosons other superpartners of the SM particles, collectively called sparticles, will inevitably be produced, if they are within the kinematic reach of the LHC. Among the large variety of SUSY events there are quite a few sharing some or all features of one or more typical Higgs signals. The following intriguing question is then, can these events obfuscate the Higgs signal? The extreme case being events from the non-Higgs sector of the MSSM faking some MSSM Higgs signals even though the latter is too weak to be observed at the LHC- at least during the early runs. It is then important to improve the purity of the Higgs signal by introducing additional selection criteria which will suppress both SM and SUSY backgrounds. Now the issue is whether these additional cuts retains most of the Higgs signal without affecting the discovery potential at the LHC.

In order to illustrate the possible impact of SUSY events on non-standard Higgs signals, we simulate the charged Higgs signal in the MSSM in the channel $g b \rightarrow t H^{-}+$c.c,$H^{-} \rightarrow$ $\tau^{-}+\nu_{\tau}$ using the event generator PYTHIA [5] along with squark-gluino events. The final state of interest is one tagged $\tau$ jet, one tagged $b$ jet and missing transverse energy $\left(\mathbb{F}_{T}\right)$. In addition we require one reconstructed $W$ and one reconstructed top quark. This signal has been studied by several groups $[2,3,4,6]$. However, the selection criteria were designed to tame the SM background alone. These analyses are, therefore, valid only if all sparticles are very heavy with negligible production cross sections at the LHC.

It is clear that SUSY events with tagged $b$ and $\tau$ jets in the final state are potentially the most dangerous BSM background. Obfuscation of the Higgs signal will be even more serious if the events contain reconstructed $t$ quarks and $W$ bosons - either genuine or fake. 
To demonstrate the interplay between the two sectors of the MSSM, we have chosen several points in the parameter space (see Section 2). Some of them are almost tailor made for mimicking the Higgs signal and we showcase our point with these examples. We then change one or more key features of the parameter space considered to show that the interplay happens over a much wider parameter space.

We have mostly worked in the unconstrained MSSM without invoking any assumption regarding the boundary conditions at a high scale (say at $M_{G}$ ). However, we have also analyzed one mSUGRA scenario [7] with $\widetilde{\tau}-\widetilde{\chi}_{1}^{0}$ coannihilation contributing to the DM relic density which turns out to be consistent with WMAP data [8]. This scenario also provides a large background to the charged Higgs signal.

In Section 3 we present a thorough analysis of the charged Higgs signal as well as the SM and SUSY backgrounds and suggest kinematical cuts suitable for obtaining pure charged Higgs events while keeping the SM and the SUSY backgrounds at the minimal level. We also briefly study the response of charged Higgs events to selection criteria for squark-gluino search.

The summary of the paper and the conclusions are in Section 4.

\section{The SUSY scenarios}

We begin with a MSSM parameter space with $m_{\tilde{q}}>m_{\tilde{g}}$, where the subscript $\tilde{q}$ refers to squarks of both L and R-types belonging to the first two generations. Thus gluino decays are dominated by the modes $\tilde{g} \rightarrow f_{3} \tilde{f}_{3}^{*}$, where $f_{3}\left(\tilde{f}_{3}\right)$ denotes a third generation quark (squark) i.e, $t$ or $b(\tilde{t}$ or $\tilde{b})$. Moreover $\tilde{f}_{3}$ is assumed to be significantly lighter than $\tilde{q}$. This is well motivated since the lighter mass eigenstates $\tilde{t}_{1}$ and $\tilde{b}_{1}$ are lighter than the $\tilde{q}$ 's in general due to mixing in the third generation squark mass matrices. This assumption is especially appropriate for the $\tilde{b}_{1}$ since $\tan \beta$ is large in scenarios with favourable production cross sections for the charged Higgs boson. Thus squark-gluino events will surely contain quite a few hard taggable $b$-jets and genuine top quarks.

In the chosen parameter space the lighter chargino $\left(\widetilde{\chi}_{1}^{ \pm}\right)$and the second lightest neutralino $\left(\widetilde{\chi}_{2}^{0}\right)$ dominantly decay into two body decay channels, $\widetilde{\chi}_{1}^{-} \rightarrow \widetilde{\tau}_{1}^{-} \nu, \widetilde{\chi}_{2}^{0} \rightarrow \widetilde{\tau}_{1} \tau$, since $\widetilde{\tau}_{1}$, like $\tilde{b}_{1}$ is also naturally lighter than the other charged sleptons $(\tilde{e}, \tilde{\mu})$ at large $\tan \beta$. This leads to an abundance of taggable $\tau$-jets in the final state. 
Throughout this paper we compute the SUSY spectra from MSSM inputs using SuSpect v2.3 [9], which takes into account radiative corrections to the sparticle masses. We fix $\tan \beta=40$ and $\mu=500 \mathrm{GeV}$. In this section all masses, mass parameters and variables with dimensions of mass are in GeV. For simplicity we assume $M_{1}$ and $M_{2}$, the $\mathrm{U}(1)$ and $\mathrm{SU}(2)$ gaugino masses, follow the relation $M_{1}=0.5 M_{2}$, which is the typical expectation in models with a unified gaugino mass in the electroweak sector at a high scale. But we assume $M_{3}$, the SU(3) gaugino mass to be a free parameter not related either with $M_{1}$ or $M_{2}$ by any unification relation. We shall, however, vary $M_{3}$ such that $M_{3}>M_{2}$ and for some choices $M_{3}$ also satisfies the unification condition.

It bears recall that in the decoupling regime ( $m_{A}$ (the pseudo scalar Higgs boson mass) $\gg m_{Z}$ ) the lighter Higgs scalar (h) has properties identical to the SM Higgs boson. It is, therefore, imperative to discover at least one more Higgs boson to establish an extended Higgs sector. The task becomes more challenging if the additional Higgs bosons are heavy. In view of this we consider several values of $m_{A}$ ranging from (500-800), which leads to approximately the same charged Higgs mass since, $m_{H^{ \pm}}^{2}=m_{A}^{2}+m_{W}^{2}$ (in the lowest order).

In the first part of our analysis we do not employ any specific model. Instead we choose general MSSM parameters constrained by general requirements like absence of flavour changing neutral currents [10], stability of the scalar potential [11] etc. To begin with the following, masses are set to fixed values:

$$
m_{\tilde{q}_{L, R}}=1000+\text { appropriate } \mathrm{D}-\text { term, } m_{\tilde{g}}=560, m_{\tilde{e}_{L, R}}=303, m_{\tilde{\nu}_{L}}=293 .
$$

From the input value of $M_{1}=150$ we obtain following masses:

$$
m_{\widetilde{\chi}_{1}^{0}}=149, m_{\widetilde{\chi}_{2}^{0}}=298, m_{\widetilde{\chi}_{1}^{+}}=298 .
$$

The masses for the 3rd generation sfermions are fixed by the following parameters:

$$
m_{t_{L}, b_{L}}=600, m_{b_{R}}=500, m_{\tau_{L}}=350, m_{\tau_{R}}=250, A_{t}=-900, A_{b}=-900, A_{\tau}=-500 .
$$

We first present our results for three choices of parameters SUSY I, II and III characterized by $m_{\tilde{t}_{R}}=350,400,453$ which along with eq. 3 lead to the spectra for the third generation sfermions in Table 1 . In Table 2 , we present the main decay channels of $\tilde{g}, \tilde{t}_{1}$ and $b_{1}$ squarks for the three scenarios in Table 1. The mass of the lighter top squarks increases as we go from SUSY I - III. This drastically affects the gluino branching ratios (BRs) and number of 


\begin{tabular}{|c|c|c|c|c|c|c|}
\hline Model & $m_{\tilde{t}_{1}}$ & $m_{\tilde{t}_{2}}$ & $m_{\tilde{b}_{1}}$ & $m_{\tilde{b}_{2}}$ & $m_{\tilde{\tau}_{1}}$ & $m_{\tilde{\tau}_{2}}$ \\
\hline SUSY I & 306 & 677 & 500 & 630 & 215 & 378 \\
\hline SUSY II & 353 & 683 & 500 & 630 & 215 & 378 \\
\hline SUSY III & 397 & 690 & 500 & 630 & 215 & 378 \\
\hline
\end{tabular}

Table 1: Masses of 3rd generation sparticles.

genuine $\mathrm{t}$ - quarks in the final state. In all cases, the dominant BRs of the $\tilde{\chi}_{2}^{0}$ and $\tilde{\chi}_{1}^{ \pm}$are practically fixed at $B R\left(\widetilde{\chi}_{1}^{-} \rightarrow \widetilde{\tau}_{1}^{-} \nu_{\tau}\right)=0.91$ and $B R\left(\widetilde{\chi}_{2}^{0} \rightarrow \widetilde{\tau}_{1} \tau\right)=0.91$. The first part of our analysis in the next section is based on SUSY I - SUSY III.

\begin{tabular}{|c|c|c|c|}
\hline Channels & SUSY I & SUSY II & SUSY III \\
\hline$\tilde{g} \rightarrow \tilde{t}_{1} t$ & 0.80 & 0.61 & - \\
$\tilde{g} \rightarrow \tilde{b}_{1} b$ & 0.18 & 0.38 & 1.0 \\
$\tilde{t}_{1} \rightarrow \tilde{\chi}_{1}^{+} b$ & 1.0 & 0.40 & 0.47 \\
$\tilde{t}_{1} \rightarrow \tilde{\chi}_{1}^{0} t$ & - & 0.60 & 0.52 \\
$\tilde{b}_{1} \rightarrow \tilde{\chi}_{1}^{0} b$ & 0.29 & 0.34 & 0.39 \\
$\tilde{b}_{1} \rightarrow \tilde{\chi}_{2}^{0} b$ & 0.26 & 0.31 & 0.35 \\
$\tilde{b}_{1} \rightarrow \tilde{\chi}_{1}^{+} t$ & 0.17 & 0.20 & 0.22 \\
$\tilde{b}_{1} \rightarrow \widetilde{t}_{1} W$ & 0.27 & 0.15 & 0.33 \\
\hline
\end{tabular}

Table 2: BR of gluinos and 3rd generation squarks.

In the next phase of the analysis we fix the masses of the third generation sfermions as follows: $m_{\tilde{b}_{1}}=755, m_{\tilde{b}_{2}}=980, m_{\tilde{t}_{1}}=751, m_{\tilde{t}_{2}}=1007, m_{\tilde{\tau}_{1}}=215, m_{\tilde{\tau}_{2}}=378$ and successively increase the gluino mass as follows, $m_{\tilde{g}}=790,950,1020,1180$ and 1345( SUSY IV - VIII) keeping all other parameters as in eq. 1 and 2. The first two choices (SUSY IV and V) correspond to $m_{\tilde{g}}<m_{\tilde{q}}$ and the gluino still decays dominantly into third generation squarks. However, the fraction of final states with genuine t quarks decreases. Hence, the probability of the SUSY events faking the $H^{ \pm}$signal should also decrease in principle. In practice, however, due to fake top reconstruction SUSY remains a potential threat to the charged Higgs signal (see Section 3). For $m_{\tilde{g}}=1020$ (SUSY VI) the squarks and gluinos are 
nearly degenerate. Finally for the last two choices $m_{\tilde{g}}>m_{\tilde{q}}$ and the gluino decays to squarkantiquark or quark-antisquark pairs belonging to the first two generations open up. This further reduces the presence of $\mathrm{b}$ and $\mathrm{t}$ in the final state. Apriorily the SUSY background is expected to be even smaller.

In addition to the above parameter spaces we also analyze the following mSUGRA point consistent with the DM data [8],

$$
m_{0}=230, m_{1 / 2}=420, A_{0}=0, \tan \beta=40, \operatorname{sign}(\mu)>0 .
$$

The resulting mass spectrum is calculated using SuSpect [9] which gives for $m_{t}=173$ :

$$
\begin{aligned}
& m_{\tilde{u}_{L}}=918, m_{\tilde{d}_{L}}=922, m_{\tilde{u}_{R}}=888, m_{\tilde{d}_{R}}=886, m_{\tilde{g}}=977, m_{\tilde{b}_{1}}=788, \\
& m_{\tilde{b}_{2}}=849, m_{\tilde{t}_{1}}=694, m_{\tilde{t}_{2}}=866, m_{\tilde{e}_{L}}=365, m_{\tilde{e}_{R}}=280, m_{\tilde{\nu}_{L}}=356, \\
& m_{\tilde{\tau}_{1}}=182, m_{\tilde{\tau}_{2}}=370, m_{\widetilde{\chi}_{1}^{0}}=171, m_{\widetilde{\chi}_{2}^{0}}=323, m_{\tilde{\chi}_{1}^{+}}=322 m_{H^{ \pm}}=500 .
\end{aligned}
$$

In this scenario the relevant $\mathrm{BRs}$ are $\mathrm{BR}\left(\tilde{g} \rightarrow t \tilde{t}_{1}\right)=20 \%, \mathrm{BR}\left(\tilde{g} \rightarrow b \tilde{b}_{1}\right)=26 \%, \operatorname{BR}\left(\tilde{\chi}_{1}^{-} \rightarrow\right.$ $\left.\widetilde{\tau}_{1}^{-} \nu_{\tau}\right)=95 \%$ and $\operatorname{BR}\left(\widetilde{\chi}_{2}^{0} \rightarrow \widetilde{\tau}_{1} \tau\right)=94 \%$. The DM relic density calculated by microOMEGAs (v 2.0) [12] yields $\Omega h^{2}=0.12$. The neutralino bulk annihilation [13, 14] contributes $37 \%$ to the relic density whereas $\widetilde{\chi}_{1}^{0}-\widetilde{\tau}$ coannihilation $[14,15]$ contributes $63 \%$.

In the following section we shall simulate the charged Higgs signal, the SM background and the SUSY events corresponding to the above choices of parameters.

\section{Simulation of the signal and the backgrounds}

At the LHC the dominant contribution to single charged Higgs production comes from the processes $g b \rightarrow t H^{-}+$c.c and $g g \rightarrow t \bar{b} H^{-}+$c.c. Both are related as they stem from gluon splitting $(g \rightarrow b \bar{b})$ inside a proton. They can, therefore, be regarded as two different approximation of the same physical process [16].

For simulating charged Higgs production with an event generator at the LHC, the process $g b \rightarrow t H^{-}+$c.c along with parton showering is considered if the additional b quark in the final state is not observable. The initial b-quark is considered as one of the five massless partons in the proton. In this approximation the $b \bar{b}$ pairs from the gluon splitting belong to the region of the phase space where both are collinear with the gluon. The resulting large 
logarithms due the massless b-quarks can be consistently absorbed into the corresponding parton density function (PDF). This gives a well defined leading order cross section.

We have simulated the leading order (LO) process,

$$
g b \rightarrow t H^{-}+c . c
$$

with the top decaying hadronically: $t \rightarrow b q \bar{q}^{\prime}$ and the charged Higgs into the $\tau-\nu_{\tau}$ channel: $H^{-} \rightarrow \tau^{-} \nu_{\tau}$. This leads to a final state consisting a single $\tau$-jet, $b$-jet accompanied by missing energy due to the neutrinos and jets. We use PYTHIA [5] to simulate the signal in eq. 6 . The cross sections are estimated setting both renormalization and as well as factorization scale, $\mu_{R}=\mu_{F}=\hat{s}$ and using CTEQ5L PDFs [17]. In the next to leading order (NLO) process the $K$ factor for the signal process is $\approx 1.5$ [16].

The dominant SM backgrounds are due to the top pair production and QCD events with jets mis-tagged as $\tau$-jets. We estimate these background along with the SUSY backgrounds arising from squark-gluino events. The LO cross section for $t \bar{t}$ is obtained using CalcHEP [18] (version 2.3.7). We require one top to decay hadronically and the other into a $\tau$ and a neutrino along with a $b$ quark.

The cross section for QCD has been computed by PYTHIA in two bins: (i) $400<\hat{p_{T}}<$ 1000 and (ii) $1000<\hat{p_{T}}<2000 \mathrm{GeV}$. The corresponding cross sections are $2041 \mathrm{pb}$ and $10 \mathrm{pb}$ respectively. The contributions from other bins are negligible. Both the above cross sections are orders of magnitude larger than the signal cross sections (see Table 3) and suitable kinematic selection will be invoked to suppress them.

Using ALPGEN [19] we have also considered the background from from $W+3$ jets , where all possible jet combinations including a $b \bar{b}$ pair and a light jet have been considered. This background, however, is not very serious (see Table 3).

In our simulation using PYTHIA we have taken into account the effects of initial and final state radiation as well as fragmentation and hadronization. A simple toy calorimeter simulation has been implemented with the following criteria:

- The calorimeter coverage is $|\eta|<4.5$ with segmentation of $\Delta \eta \times \Delta \phi=0.09 \times 0.09$ which resembles a generic LHC detector.

- A fixed cone algorithm with $\Delta R=\sqrt{\Delta \eta^{2}+\Delta \phi^{2}}=0.5$ has been used for jet finding.

- Jets are ordered in $E_{T}$ with $E_{T, \text { min }}^{\text {jet }}=20 \mathrm{GeV}$. 
b- jet identification:

We have tagged $b$-jets in our analysis by the following procedure. A jet with $|\eta|<2.5$ corresponding to the coverage of tracking detectors matching with a $B$-hadron of decay length $>0.9 \mathrm{~mm}$ has been marked tagged. This criteria ensures that single $b$-jet tagging efficiency (i.e., the ratio of tagged $b$-jets and the number of taggable $b$-jets) $\epsilon_{b} \approx 0.5$ in $t \bar{t}$ events.

$\underline{\tau-\text { jet identification: }}$

Taus are identified through their hadronic decays producing narrow jets with 1 or 3 tracks pointing to the jets. We have defined a narrow signal cone of size $\Delta R_{S}=0.1$ and an isolation cone of size $\Delta R_{I}=0.4$ around the calorimetric jet axis. We then require 1 or 3 charged tracks inside the signal cone with $\left|\eta_{\text {track }}\right|<2.5$ and $P_{T}>3 \mathrm{GeV}$ for the hardest track. We further require that there are no other charged tracks with $P_{T}>1 \mathrm{GeV}$ inside the isolation cone to ensure tracker isolation.

Top quark reconstruction:

We reconstruct one top quark following the procedure of [20] summarized below. First we compute the invariant mass of any two jets which are not tagged as $b$-jets or $\tau$-jets, to reconstruct a candidate $W$. Further each tagged $b$-jet is combined with a candidate $W$ to obtain a candidate top quark. For each candidate a $\chi_{\text {top }}^{2}$ is defined:

$$
\chi_{\text {top }}^{2}=\left(\frac{m_{W}-m_{W}^{r e c}}{15}\right)^{2}+\left(\frac{m_{t}-m_{t}^{r e c}}{25}\right)^{2}
$$

where $m_{W}=80.42 \mathrm{GeV}$ and $m_{t}=173.1 \mathrm{GeV}$ are world averages of $W$-boson and top quark masses and $m_{W}^{r e c}$ and $m_{t}^{r e c}$ are reconstructed masses of $W$-boson and top quark candidates respectively. We have implemented $\chi^{2}$ minimization procedure assuming a spread of $15 \mathrm{GeV}$ and $25 \mathrm{GeV}$ for the reconstructed $W$ and top candidates respectively. These numbers have been determined using Monte Carlo (MC) informations of jets originating from the decays of $W$-boson and top quarks respectively.

Finally from MC information we have required minimum value of $\chi^{2}$ for an acceptable event to be less than 8 . This choice yields good efficiency for the reconstructed top in $t \bar{t}$ event.

The following selection criteria (SC) similar to those used in [21] have been used for rejection of the SM background:

- We have required only one tagged $b$-jet (Cut 1 ). 
- We have asked for one identified $\tau$-jet (Cut 2).

- Events should have one detected $\tau$-jet with $E_{T}^{\tau-j e t}>100 \mathrm{GeV}$ (Cut 3).

- Events should have missing transverse energy $E_{T}>100 \mathrm{GeV}$ (Cut 4).

- We require at least three jets in addition to one extra $\tau$ jet in the event (Cut 5).

- We require one reconstructed top as described above (Cut 6).

- We have also investigated the azimuthal opening angle in the transverse plane between the $\tau$-jet and the $E_{T}$ vector. We have selected events with $\Delta \phi\left(\tau-j e t, E_{T}\right)>60^{\circ}$ (Cut $7)$.

Table 3 shows the cumulative efficiencies of the Cuts 1-7 for $m_{H^{ \pm}}=500,600,700,800$ $\mathrm{GeV}$ and $\tan \beta=40$ along with the $t \bar{t}$ and QCD backgrounds. The $\mathrm{W}+$ jets backgrounds are not shown as they are negligible. Notice that in all cases except for the QCD background background, about $50 \%$ of events have only one tagged $b$-jet. Moreover, for all $m_{H^{ \pm}}$, the overall efficiencies of the cuts for the signal are roughly the same $(\sim 5-6 \%)$, where as for the background it is about $0.015 \%$. The $t \bar{t}$ background is mostly killed by the strong cut on the $E_{T}$ of the $\tau$-jets as the $\tau$-jets originating from the charged Higgs in the decoupling regime are indeed much harder. The final cross section $\times$ efficiency $\left(\epsilon_{1}\right)$ for all types of events after Cuts (1-7) are also presented (the appropriate BRs are included in $\epsilon_{1}$ ). They lie in the range $0.8-4.2 \mathrm{fb}$. The corresponding number for the total SM background is $23.5 \mathrm{fb}$.

As is well-known, the above cuts are inadequate to establish the charged Higgs signal, even if the SUSY background is negligible. For example, corresponding to $m_{H^{ \pm}}=500(700)$ $\mathrm{GeV}$ which yields the largest (the third largest) signal cross section the significance $\left(\frac{S}{\sqrt{B}}\right)$ is 4.75 (1.58) for $\mathcal{L}_{\text {int }}=30 \mathrm{fb}^{-1}$.

In Table 4 we present the cross sections and efficiencies for squark-gluino events computed by [18] for the three model parameter spaces in Table 1 , subject to the same set of cuts. It should be borne in mind that the SUSY spectrum are the same for the three sets except for $m_{\tilde{t}_{1}}$. We note that the final cross section of the SUSY events is significant in all cases. In fact they are comparable to the $t \bar{t}$ background and even the weakest among them is larger than the signal for all $m_{H^{ \pm}}$.

As discussed in Section 2, the number of genuine top quarks in SUSY III events is much smaller compared to the ones in SUSY I and SUSY II. Yet we find from Table 4 that the 
efficiency of getting one reconstructed top candidate is fairly high for all the SUSY scenarios under consideration. This implies that due to combinatorial backgrounds significant number of fake top candidates are being reconstructed even in SUSY III.

We have also repeated the analysis using the $t$-reconstruction prescription of [21]. Our method yields better top reconstruction efficiency for the $H^{ \pm}$signal. However, the procedure of [21] also yields sizable SUSY backgrounds via fake t-quarks in squark-gluino events.

The simultaneous presence of the Higgs and SUSY events leads to several interesting conclusions although some of them could be misleading, as we shall see below. After the first set of Cuts (1-7) together they can show up as a clear indication of BSM physics standing over the SM background although, as already noted, the Higgs events by themselves are not statistically significant. It is also important to note that this will also disfavour a two Higgs doublet extension of the SM with a charged Higgs of comparable mass and BRs.

It follows from Table 3 and 4 that for $m_{H^{ \pm}}=500 \mathrm{GeV}$ and SUSY I the significance of the combined BSM signal is $7.5(13)$ for $\mathcal{L}_{\text {int }}=1(10) \mathrm{fb}^{-1}$. If we consider the smallest contribution from the SUSY events (SUSY III), the significance is still 5.8 at $\mathcal{L}_{\text {int }}=10 \mathrm{fb}^{-1}$. Thus the BSM physics can be established at early stages of the LHC experiment although the final state will be an admixture of Higgs and squark-gluino events.

For $m_{H^{ \pm}}=500$ (800) GeV and SUSY I (III) Higgs events can be as large as $27 \%$ $(17 \%)$ of the number of SUSY events. However, there are cases where the SUSY events simply dwarf the tiny presence of the Higgs. Consider, for example, SUSY II and $m_{H^{ \pm}}=800$ $\mathrm{GeV}$, which corresponds to the weakest Higgs signal. Nevertheless, the significance of the combined signal is 6.5 for $\mathcal{L}_{i n t}=10 \mathrm{fb}^{-1}$. This example clearly demonstrates that even if the Higgs signal is negligible, SUSY events alone can masquerade as the charged Higgs signal. Additional selection criteria are, therefore, called for to disentangle the two types of events and confirm the Higgs signal.

It is known for a long time that the kinematic distribution of the decay products of polarized $\tau$-leptons can be exploited in new physics search $[22,23]$. In conjunction with the standard cuts (Cuts $1-7$ ), $\tau$-polarization is very effective in suppressing the SM backgrounds relative to the charged Higgs signal (for both $m_{H^{ \pm}}<m_{t}$ and $m_{H^{ \pm}}>m_{t}$ ) in the $\tau-\nu_{\tau}$ channel [22]. The SM background being suppressed is mainly due to the decay from $W \rightarrow \tau \nu_{\tau}$. The main reason is that the polarization of the $\tau^{\prime}$ 's in the charged Higgs decay $\left(P_{\tau}=+1\right)$ and $W$ decay are opposite. As a result the decay products of the $\tau$ 's originating from the charged 
Higgs have very different energy distribution than their counterparts stemming from W-decay . In fact most of the subsequent analyses [3, 4, 21, 24] have exploited this feature to improve the significance of the Higgs signal. In addition it also eliminates the QCD background very effectively.

However, cuts based on $\tau$-polarization may not be very efficient in presence of SUSY backgrounds which is at the focus of interest of this paper. The energetic $\tau$ 's in the squarkgluino decay cascades, which passes the selection criteria, mostly arise from the decays of $\widetilde{\tau}_{1}^{\prime}$ 's $\left(\widetilde{\tau}_{1} \rightarrow \tau \widetilde{\chi}_{1}^{0}\right)$. The polarization of these $\tau$ 's may have a wide variety depending on the composition of $\widetilde{\tau}_{1}$ and the lightest supersymmetric particle (LSP) [25]. For example, if $\widetilde{\tau}_{1}$ is dominantly a $\widetilde{\tau}_{R}$ and the LSP is bino like, the polarization of a $\tau$ in SUSY cascades will be identical to that of a $\tau$ arising from charged Higgs decay. This is true for all the parameter spaces considered by us. In fact it has already been noted [26] that the polarization of the $\tau$ 's in the squark-gluino decay cascades are dominantly with $P_{\tau} \approx+1$ in the mSUGRA type of model. This property enhances the SUSY signal in the interesting region of parameter space where LSP- $\widetilde{\tau}_{1}$ coannihilation [15] can generate the DM relic density of the universe. In general, however, the $\widetilde{\tau}_{1}$ may be an admixture of both $\widetilde{\tau}_{L}$ and $\widetilde{\tau}_{R}$. But even in this case a significant fraction of the $\tau$ 's will pass the selection criteria.

The observations in the last paragraph can be justified by the sparticle spectra and BRs presented in the last section. For example in SUSY I $\tilde{t}_{1} \widetilde{t}_{1}^{*}$ pair production has a significant cross section $(3.3 \mathrm{pb})$ in SUSY I. The $\tilde{t}_{1}$ decays into $b \tilde{\chi}_{1}^{+}$with $100 \%$ BR. The $\tilde{\chi}_{1}^{+}$dominantly decays into $\widetilde{\tau}_{1}^{+} \nu_{\tau}(\mathrm{BR}=91 \%)$. Thus the highly energetic $\tau^{\prime}$ 's in this decay chain come from $\widetilde{\tau}_{1}$ decay. A small fraction of the $\tau$ s come from the decay of $\widetilde{\chi}_{1}^{+} \rightarrow \widetilde{\nu}_{\tau} \tau^{+}$followed by the invisible decay of $\widetilde{\nu}_{\tau}$ into $\nu_{\tau} \widetilde{\chi}_{1}^{0}$. These $\tau$ 's are rather soft because of the small mass difference between $\widetilde{\chi}_{1}^{+}$and $\widetilde{\nu}_{\tau}$ and fails to survive the strong cut on the $E_{T}$ of the $\tau$-jet.

Gluino pair production also has a large cross section $(4.6 \mathrm{pb})$. From Table 2 it follows that gluino decays dominantly into $\tilde{t}_{1} t$ pairs. As already discussed energetic $\tau$ 's will come from $\widetilde{\tau}_{1}$ decay cascade. The $\tau$ 's from W-decay are killed by the strong $E_{T}$ cut (Cut 3 ).

Of course a small fraction (18\%) of the gluinos decay into $\widetilde{b}_{1} b$. From Table 2 we can see $\widetilde{b}_{1}$ decays into $\widetilde{\chi}_{2}^{0} b$ with $\operatorname{BR}(26 \%)$. Here $\widetilde{\chi}_{2}^{0}$ decays into $\widetilde{\tau}_{1} \tau$ with large $\operatorname{BR}(90 \%)$. Most of the primary $\tau$ in these decays are removed by Cut 3 as already noted. The secondary $\tau$ 's from $\widetilde{\tau}_{1}$ follows the polarization pattern as noted above. Similarly the decay chain $\widetilde{b}_{1} \rightarrow \widetilde{\chi}_{1}^{-} t$ yield energetic $\tau$ 's with polarization properties as noted above. The other significant decay modes 
of $\widetilde{b}_{1}\left(\widetilde{\chi}_{1}^{0} b\right.$ and $\left.\widetilde{t}_{1} W\right)$ either yield no $\tau^{\prime}$ 's or dominantly positively-polarized $\tau$ 's.

In SUSY I the squarks of first two generations decay mainly into quark- gluino pairs. For example, $\tilde{u}_{L}$ decays primarily into $\tilde{g} u$ with $\mathrm{BR}(78 \%)$ and $\tilde{\chi}_{1}^{+} d$ with $\operatorname{BR}(13 \%)$. As elaborated above the gluino and chargino decays will mainly lead to $\tau$ 's with $P_{\tau}=+1$. The $\tilde{u}_{R}$ decays primarily into $\tilde{g} u$ with $\operatorname{BR}(95 \%)$ and $\widetilde{\chi}_{1}^{0} u$ with $\operatorname{BR}(5 \%)$. Thus energetic $\tau^{\prime}$ s in the squark decay chains will also yield right handed polarized jets.

Applying similar chains of arguments to other SUSY scenarios (SUSY II -VIII) considered in this paper, it is easy to see that in all cases the required energetic $\tau$-jets dominantly come from $\widetilde{\tau}_{1}$ decays and has $P_{\tau} \approx 1$.

To roughly estimate the possible impact of the SUSY background on the Higgs signal, we note from Tables 1 and 2 of [21] that the significance of the signal for $m_{H^{ \pm}}=600 \mathrm{GeV}$ against a SM background of $0.22 \mathrm{fb}$ after Cuts 1-7 and the $\tau$-polarization cut is 5.6 at $\mathcal{L}_{i n t}=30 \mathrm{fb}^{-1}$. If we assume that approximately all ( $50 \%$ ) of the weakest background from SUSY III is retained after the $\tau$-polarization cut, the significance reduces to 1.2 (1.6) for 30 $\mathrm{fb}^{-1}$.

In lieu of $\tau$-polarization we,therefore, add two more generic cuts which does not depend on the compositions of the sparticles. Instead they essentially depend on the kinematics of the decay of a heavy Higgs. In order to motivate these cuts we present in Fig. 1 the distributions of the $E_{T}$ of the $\tau$-jets and $\mathbb{F}_{T}$ (after Cuts 1-7) in the Higgs, SUSY and SM events. Fig. 1 suggests that a stronger $\mathbb{E}_{T}$ cut removes the SM backgrounds efficiently while the stronger cut on the $\tau$-jet $E_{T}$ suppresses both SM and SUSY backgrounds, while retaining bulk of the signal.

The new kinematical selections are:

- A more stingent cut on $\tau$-jet momentum, $E_{T}^{\tau-j e t}>180 \mathrm{GeV}$ (Cut 8).

- A stronger $\mathbb{F}_{T}$ cut, $\mathbb{F}_{T}>260 \mathrm{GeV}(\mathrm{Cut} 9)$.

We present the cross sections after Cuts 8 and 9 for the signal(Table 3) along with the SM (Table 3) and the SUSY backgrounds(Table 4). In all cases $\epsilon_{2}$ is the combined efficiency of the Cuts 1-9. The QCD background is eliminated. The $t \bar{t}$ background is $\sim$ 2.1 for $\mathcal{L}_{i n t}=30 \mathrm{fb}^{-1}$. The number of signal events for this $\mathcal{L}_{i n t}$ for $m_{H^{ \pm}}=500,600,700$ and $800 \mathrm{GeV}$ are 8.1, 14.1, 13.5 and 10.2 respectively. In the absence of SUSY backgrounds these cuts are adequate for establishing the charged Higgs signal for $m_{H^{ \pm}}>500 \mathrm{GeV}$. For 

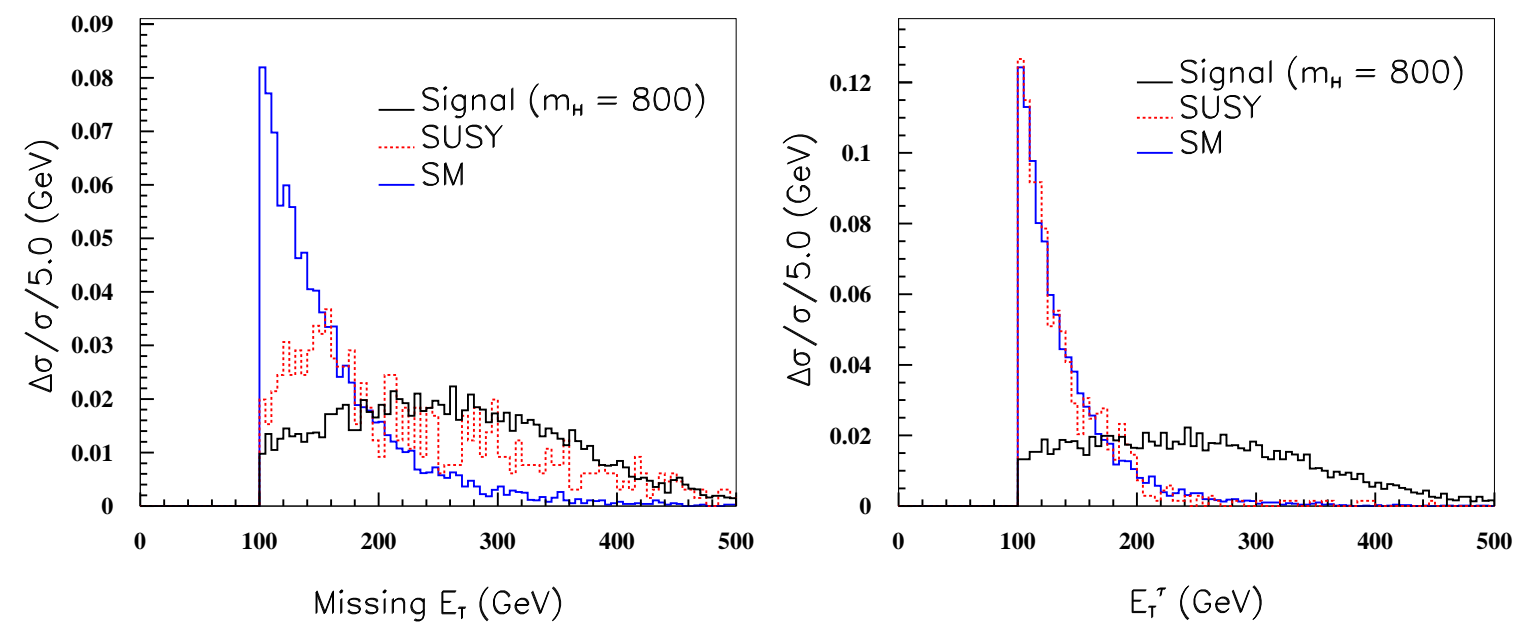

Figure 1: The distributions (normalised to unity) for signal (blue), SUSY (red) and SM (black) of $\mathbb{F}_{T}$ (left) and $E_{T}^{\tau-j e t}$ (right) after selection Cuts $1-7$. Here $m_{H^{ \pm}}=800 \mathrm{GeV}$.

$m_{H^{ \pm}} \lesssim 500 \mathrm{GeV}$ higher luminosity may be required inspite of the larger production cross section, since the Cuts 8 and 9 are less efficient. We finally note that this generator level analysis indicates that the reach in $m_{H^{ \pm}}$, in the absence of SUSY backgrounds, is at least as good as that obtained in earlier works, if not better.

It also follows from Tables 3 and 4 that the new cuts improve the fraction of Higgs event compared to the SUSY events in the combined BSM signal. For example, corresponding to $m_{H^{ \pm}}=500$ (800) GeV and SUSY I (III), Higgs events can be as large as 32\% (180\%) of the number of SUSY events. The modest increase of the signal relative to the SUSY background for $m_{H^{ \pm}}=500 \mathrm{GeV}$, suggest that this selection procedure though very effective in the decoupling regime may not work for a lighter charged Higgs.

However, even after the new cuts the Higgs signal is substantially contaminated by the squark gluino events in some cases. For example the significance of the Higgs signal for $m_{H^{ \pm}}=600 \mathrm{GeV}$ and SUSY I (III) is 2.7 (5) at $\mathcal{L}_{i n t}=30 \mathrm{fb}^{-1}$ when the total background ( $\mathrm{SM}+\mathrm{SUSY}$ ) is taken into account.

To increase the purity of the Higgs signal we further require that

- $N_{\text {jet }} \leq 6$, where, $N_{\text {jet }}$ is the number of jets in an event (Cut 10). 


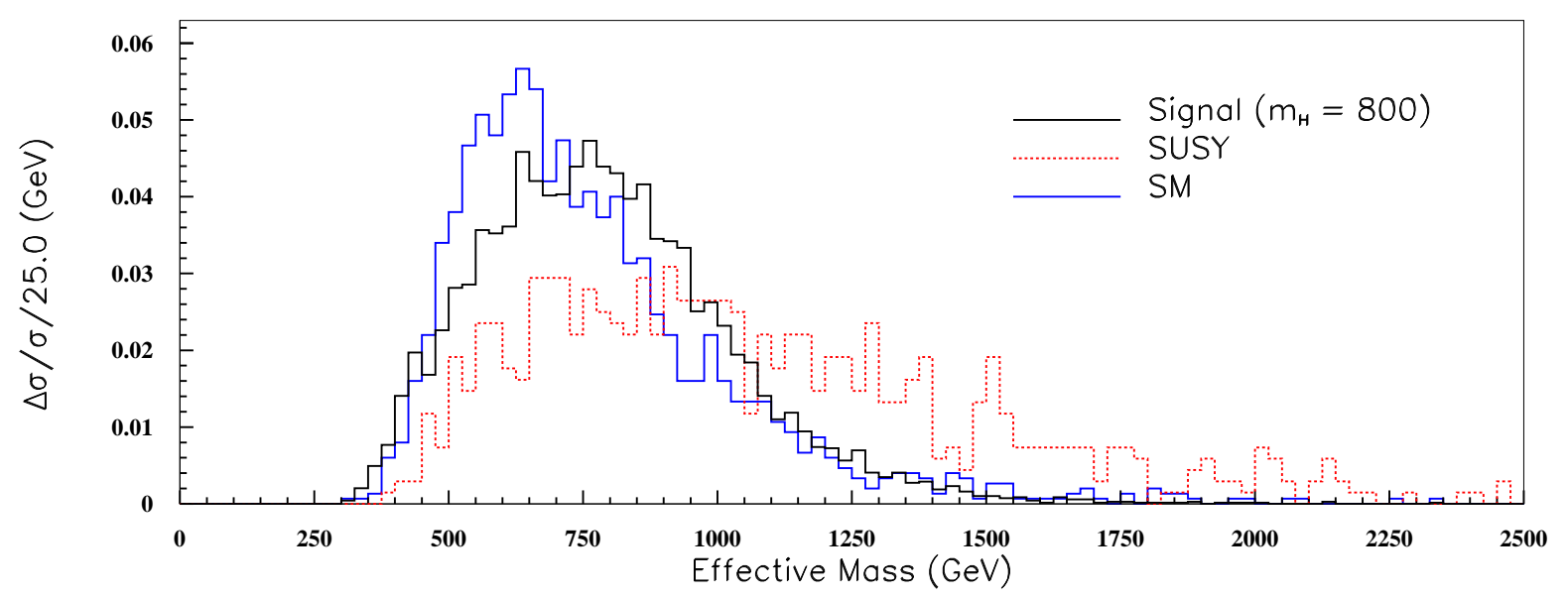

Figure 2: The distributions (normalised to unity) for signal (blue), SUSY (red) and SM (black) of $M_{e f f}$ after Cuts 1-7.

This cut reflects the fact that the jet multiplicity in SUSY events is in general larger than that in the Higgs signal. In Tables 3 and 4, the total efficiency after Cuts 1-10 is presented by $\epsilon_{3}$. Cut 10 practically eliminates the $t \bar{t}$ events but some SUSY backgrounds remain (see Tables 3 and 4).

We present in Table 5 the significance for different $m_{H^{ \pm}}$taking into account the largest SUSY background (SUSY I) at $\mathcal{L}_{\text {int }}=30 \mathrm{fb}^{-1}$. The signal is observable for $600 \lesssim m_{H^{ \pm}} \lesssim 800$ $\mathrm{GeV}$. For $m_{H^{ \pm}}=500 \mathrm{GeV}$ the signal will be observable at $\mathcal{L}_{\text {int }}=50 \mathrm{fb}^{-1}$. This again shows that this search strategy is more potent for $m_{H^{ \pm}}$in the deep decoupling region. We shall comment on lower Higgs masses in the following.

The number of jets in an event depend on the parton showering model of PYTHIA. This has not been tested in the LHC enviornment. We have, therefore, attempted some other selection criteria and combination thereof.

Since the signal is not expected to have many isolated hard leptons $(\mathrm{e}, \mu)$, one can veto events with a lepton with $P_{T}^{e, \mu}>15 \mathrm{GeV}$ and $|\eta| \leq 2.4$ having $\Delta R_{\text {(jet, lepton })} \geq 0.5$. However this does not improve the significance of the signal, see Table 5 .

In Fig. 2 we present the distribution of $M_{\text {eff }}$, where $M_{\text {eff }}=\left|\mathscr{E}_{T}\right|+\Sigma_{i}\left|P_{T}^{l_{i}}\right|+\Sigma_{i}\left|P_{T}^{j_{i}}\right|$ $(l=e, \mu)$, for Higgs, SUSY and SM events. Fig. 2 suggests that a cut $M_{e f f}<800 \mathrm{GeV}$ 


\begin{tabular}{|c|c|c|c|c|c|c|c|c|}
\hline & & \multicolumn{4}{|c|}{ Signal $m_{H^{ \pm}}(\mathrm{GeV})$} & \multirow[t]{2}{*}{$t \bar{t}$} & \multirow[t]{2}{*}{ QCD } & \multirow[t]{2}{*}{$W+3 j$} \\
\hline & & 500 & 600 & 700 & 800 & & & \\
\hline & $\sigma(\mathrm{pb})$ & 0.67 & 0.36 & 0.20 & 0.12 & 492 & 2042 & 46.65 \\
\hline & Selections & & & & & & & \\
\hline Cut 1 & $1 b-j e t$ & 0.539 & 0.540 & 0.545 & 0.546 & 0.486 & 0.0745 & 0.266 \\
\hline Cut 2 & $1 \tau-j e t$ & 0.210 & 0.216 & 0.219 & 0.218 & 0.112 & 0.0017 & 0.005 \\
\hline Cut 3 & $\begin{array}{l}E_{T}^{\tau-j e t}>100 \\
\mathrm{GeV}\end{array}$ & 0.142 & 0.158 & 0.169 & 0.172 & 0.0086 & $3.7 \times 10^{-5}$ & $4.6 \times 10^{-4}$ \\
\hline Cut 4 & $E_{T}>100 \mathrm{GeV}$ & 0.118 & 0.137 & 0.152 & 0.157 & 0.0023 & $7.7 \times 10^{-5}$ & $9.5 \times 10^{-5}$ \\
\hline Cut 5 & $\begin{array}{l}N_{\text {jet }} \quad \geq \\
3 \text { (except } \tau \text {-jet })\end{array}$ & 0.076 & 0.089 & 0.098 & 0.102 & 0.0021 & $6.1 \times 10^{-5}$ & $8.2 \times 10^{-5}$ \\
\hline Cut 6 & $\begin{array}{l}1 \quad \text { recon- } \\
\text { structed top }\end{array}$ & 0.054 & 0.061 & 0.066 & 0.069 & 0.0015 & $9.0 \times 10^{-6}$ & $2.1 \times 10^{-5}$ \\
\hline \multirow[t]{2}{*}{ Cut 7} & $\begin{array}{l}\Delta \phi(\tau \quad- \\
\left.\text { jet, } \mathscr{F}_{T}\right)>60^{\circ}\end{array}$ & 0.051 & 0.058 & 0.064 & 0.067 & $1.5 \times 10^{-4}$ & $6.0 \times 10^{-6}$ & - \\
\hline & $\sigma \times \epsilon_{1}(\mathrm{fb})$ & 4.2 & 2.5 & 1.4 & 0.8 & 11.2 & 12.3 & - \\
\hline Cut 8 & $\begin{array}{l}E_{T}^{\tau-j e t}>180 \\
\mathrm{GeV}\end{array}$ & 0.025 & 0.036 & 0.045 & 0.050 & $2.2 \times 10^{-5}$ & - & - \\
\hline \multirow[t]{2}{*}{ Cut 9} & $E_{T}>260 \mathrm{GeV}$ & 0.0033 & 0.011 & 0.020 & 0.028 & $1.0 \times 10^{-6}$ & - & - \\
\hline & $\sigma \times \epsilon_{2}(\mathrm{fb})$ & 0.27 & 0.47 & 0.45 & 0.34 & 0.07 & - & - \\
\hline \multirow[t]{2}{*}{$\begin{array}{l}\text { Cut } \\
10\end{array}$} & $N_{\text {jet }} \leq 6$ & 0.0028 & 0.0095 & 0.0181 & 0.0251 & - & - & - \\
\hline & $\sigma \times \epsilon_{3}(\mathrm{fb})$ & 0.24 & 0.41 & 0.40 & 0.30 & - & - & - \\
\hline
\end{tabular}

Table 3: The signal for different $m_{H^{ \pm}}$at $\tan \beta=40$ and the SM backgrounds subjected to different selection criteria.

would further suppress the SUSY background. This affects the signal to a extent larger than Cut 10 but practically eliminates the SUSY background. The corresponding number of background free signal events at $\mathcal{L}_{\text {int }}=30 \mathrm{fb}^{-1}$ have been presented in Table 5 and does not look very attractive. 
A tighter cut $N_{\text {jet }} \leq 5$ gives a more promising background free signal size. This looks even more attractive if the next to leading order cross section with a $K=1.5$ is used. Similar results have been obtained by combining the lepton veto and $N_{\text {jet }} \leq 6$ criteria.

\begin{tabular}{|l|l|l|l|l|}
\hline & & SUSY I & SUSY II & SUSY III \\
\hline & $\sigma(\mathrm{pb})$ & 12.2 & 10.1 & 8.9 \\
\hline & $m_{\tilde{t}_{1}}(\mathrm{GeV})$ & 306 & 353 & 398 \\
\hline \hline Cut 1 & Selection Criteria & & & \\
Cut 2 & $1 \tau-j e t$ & 0.32982 & 0.31205 & 0.24578 \\
Cut 3 & $E_{T}^{\tau-j e t}>100 \mathrm{GeV}$ & 0.00384 & 0.00379 & 0.00381 \\
Cut 4 & $E_{T}>100 \mathrm{GeV}$ & 0.00322 & 0.00294 & 0.00323 \\
Cut 5 & $N_{\text {jet }} \geq 3($ except $\tau$-jet $)$ & 0.00308 & 0.00281 & 0.00309 \\
Cut 6 & 1 reconstructed top & 0.00220 & 0.00204 & 0.00166 \\
Cut 7 & $\Delta \phi\left(\tau-j e t, \mathscr{F}_{T}\right)>60^{\circ}$ & 0.00129 & 9.1 & 8.5 \\
& & & $\times 10^{-4}$ & $\times 10^{-4}$ \\
\hline & $\sigma \times \epsilon_{1}(\mathrm{fb})$ & 15.7 & 9.2 & 4.7 \\
\hline Cut 8 & $E_{T}^{\tau-j e t}>180 \mathrm{GeV}$ & $2.0 \times 10^{-4}$ & $1.9 \times 10^{-4}$ & $1.3 \times 10^{-4}$ \\
Cut 9 & $E_{T}>260 \mathrm{GeV}$ & $7.0 \times 10^{-5}$ & $8.0 \times 10^{-5}$ & $3.0 \times 10^{-5}$ \\
\hline & $\sigma \times \epsilon_{2}(\mathrm{fb})$ & 0.85 & 0.80 & 0.19 \\
\hline Cut 10 & $N_{\text {jet }} \leq 6$ & $1.0 \times 10^{-5}$ & $1.0 \times 10^{-5}$ & $1.0 \times 10^{-5}$ \\
\hline & $\sigma \times \epsilon_{3}(\mathrm{fb})$ & 0.12 & 0.10 & 0.09 \\
\hline
\end{tabular}

Table 4: The SUSY background for the sample points in Table 1.

We next increase $m_{\widetilde{\tau}_{1}}$ to $275 \mathrm{GeV}$ ( the earlier choice was $215 \mathrm{GeV}$ ) and re-calculate the SUSY background keeping the other parameters in SUSY I fixed. There is a treadoff between the reduction in the number of final states with $\tau^{\prime}$ s (e.g., $B R\left(\widetilde{\chi}_{1}^{+} \rightarrow \widetilde{\tau}_{1}^{+} \nu_{\tau}\right.$ ) now becomes 0.60 only) and the increased efficiency of Cut 8. Finally, however, the SUSY background increases substantially compared to SUSY I ( $\left.\sigma \times \epsilon_{3}=0.37 \mathrm{fb}\right)$. Thus SUSY remains a potentially dangerous background for a wide variety of $m_{\widetilde{\tau}_{1}}$, as long as the lighter electroweak gauginos decay with large BRs into final states involving hard, taggable $\tau$-jets. 


\begin{tabular}{|l|l|l|l|l|}
\hline \multirow{2}{*}{ Cuts } & \multicolumn{5}{|c|}{$m_{H^{ \pm}}(\mathrm{GeV})$} \\
\cline { 2 - 5 } & 500 & 600 & 700 & 800 \\
\hline & \multicolumn{5}{|c|}{$S / \sqrt{B}$} \\
\hline$N_{\text {jet }} \leq 6$ & 4.0 & 6.5 & 6.3 & 5.0 \\
$N_{\text {lep }}=0$ & 2.0 & 3.6 & 3.4 & 2.5 \\
\hline & \multicolumn{4}{|c|}{ Background free number of signal events } \\
\hline$M_{\text {eff }}<800$ & 5 & 7 & 5 & 3 \\
$\mathrm{GeV}$ & & & & \\
$N_{\text {jet }} \leq 5$ & 6 & 10 & 10 & 7 \\
$N_{\text {lep }}=0$ and & 6 & 11 & 10 & 7 \\
$N_{\text {jet }} \leq 6$ & & & & \\
\hline
\end{tabular}

Table 5: The significance of the Higgs signal or the number of background free signal events for different selection criteria at $\mathcal{L}_{i n t}=30 \mathrm{fb}^{-1}$. The background is SUSY I.

So far our analyses were based on leading order cross sections for the signal and the backgrounds. It is worthwhile to estimate the possible impact of NLO cross sections. As already noted the K-factor for the charged Higgs signal in the NLO is 1.5 [16]. The corresponding number for the $t \bar{t}$ is $\approx 1.6[27]$. Assuming that the efficiencies do not change drastically at the NLO, the significance of the Higgs signal with respect to the SM increases slightly after Cuts 8 and 9 (compare with Table 3). The incorporation of the K-factor for squark-gluino production which, in this case is $1.3-1.4$ [28], may marginally increase the final significance of the signal vis a vis the SUSY background in Table 5.

We now present the SUSY backgrounds for different gluino masses (see Section 2) with a motivation to probe the $m_{\tilde{g}}^{\max }$ beyond which the SUSY contamination in the $H^{ \pm}$signal is negligible. In Table 6, we display the raw cross sections and as well as effective cross sections $\left(\sigma \times \epsilon_{i}\right)$. The first two cases, SUSY IV and V stand for $m_{\tilde{g}}<m_{\tilde{q}}$ where as last three scenarios, SUSY VI, VII, VIII correspond to $m_{\tilde{g}} \gtrsim m_{\tilde{q}}$. Obviously the cross section and number of genuine top quarks in the final states decreases with increasing gluino mass since $\tilde{g} \rightarrow q \tilde{q}$ channel open up with substantial branching fraction. As a result the size of the SUSY background decreases with increasing gluino mass. Yet in all cases SUSY remains the dominant background after Cut 9. After applying Cut 10 however, the SUSY background 
is significantly reduced. Again for $600 \lesssim m_{H^{ \pm}} \lesssim 800 \mathrm{GeV}$ the significance is $\gtrsim 5$ in all SUSY scenarios (IV -VIII).

\begin{tabular}{|c|c|c|c|c|c|}
\hline & SUSY IV & SUSY V & SUSY VI & SUSY VII & SUSY VIII \\
$m_{\tilde{g}}(\mathrm{GeV})$ & 790 & 950 & 1020 & 1180 & 1345 \\
$\sigma(\mathrm{pb})$ & 5.6 & 3.8 & 0.88 & 0.61 & 0.49 \\
\hline$\sigma \times \epsilon_{1}(\mathrm{fb})$ & 5.9 & 3.1 & 1.5 & 0.60 & 0.38 \\
$\sigma \times \epsilon_{2}(\mathrm{fb})$ & 0.34 & 0.30 & 0.31 & 0.12 & 0.11 \\
$\sigma \times \epsilon_{3}(\mathrm{fb})$ & 0.11 & 0.038 & 0.079 & 0.037 & 0.049 \\
\hline
\end{tabular}

Table 6: The SUSY backgrounds for increasing $m_{\tilde{g}}$ (see text for the details).

As already noted earlier the above search stratrgy may not be the best for $m_{H^{ \pm}} \lesssim 500$ $\mathrm{GeV}$. The stronger Cuts (8 and 9) may highly suppress the signal. We suggest that the earlier procedure $[2,3,4,6]$ based on the polarization of the $\tau$-jets may be applied to suppress the SM background. However, this could leave behind a large SUSY background depending on the polarization property of $\tau$ 's appearing in the SUSY cascade decays. A cut like $N_{\text {jet }} \leq 6$ could then be implemented for bringing the SUSY background under control. It is already shown in Table 3 that one can implement this cut without paying too much price for the signal.

Finally in Table 7 the result for a mSUGRA point motivated by the DM data point is presented. Since $m_{H^{ \pm}}=500 \mathrm{GeV}$ in this case the significance of the signal is rather modest as expected. We find the significance at $\mathcal{L}_{\text {int }}=30(100) \mathrm{fb}^{-1}$ is $3.4(6.2)$.

\begin{tabular}{|c|c|}
\hline & mSUGRA point \\
\hline$\sigma \times(\mathrm{pb})$ & 1.5 \\
\hline$\sigma \epsilon_{1}(\mathrm{fb})$ & 1.35 \\
$\sigma \times \epsilon_{2}(\mathrm{fb})$ & 0.45 \\
$\sigma \times \epsilon_{3}(\mathrm{fb})$ & 0.15 \\
\hline
\end{tabular}

Table 7: The SUSY background for a mSUGRA point motivated by DM data (see Section 2). 


\begin{tabular}{|c|c|c|c|c|}
\hline & Selection Criteria & $0 l$ & $1 l$ & $1 \tau+X$ \\
\hline & Before all Cuts & 0.732 & 0.240 & 0.124 \\
Cut $1^{\prime}$ & $E_{T}^{\text {jet1,jet } 2}>150 \mathrm{GeV}$ & 0.063 & 0.039 & 0.065 \\
Cut $2^{\prime}$ & $\mathbb{E}_{T}>200 \mathrm{GeV}$ & 0.084 & 0.110 & 0.197 \\
Cut $3^{\prime}$ & $M_{e f f}>1000 \mathrm{GeV}$ & 0.437 & 0.471 & 0.350 \\
Cut $4^{\prime}$ & Transverse sphericity $>0.2$ & 0.414 & 0.541 & 0.428 \\
\hline & $\sigma \times \epsilon_{4}(\mathrm{fb})$ & 2.03 & 0.75 & 0.69 \\
\hline
\end{tabular}

Table 8: The effective cross sections of $0 l, 1 l$ and $1 \tau+X$ events subjected to Cuts $\left(1^{\prime}-4^{\prime}\right)$ for $m_{H^{ \pm}}=300 \mathrm{GeV}$.

We now address the inverse problem. Namely, how the charged Higgs events may affect the canonical $m$-leptons $+n$-jets $+\mathbb{E}_{T}$ signatures of squark-gluino production. We have restricted ourselves to $m=0,1$.

In our simulation leptons $(l=e, \mu)$ are selected with $\mathrm{P}_{\mathrm{T}} \geq 30 \mathrm{GeV}$ and $|\eta| \leq 2.5$. For lepton-jet isolation we require $\Delta R(l, j)>0.5$. The detection efficiency of the leptons are assumed to be $100 \%$ for simplicity.

We have also looked into final states of the type $1 \tau+X$ where $X$ includes two or more hard jets but no $e$ or $\mu$ or tagged $\tau$. Tagging of $\tau$ jets are implemented according to the following procedure.

Only hadronic $\tau$ decays are selected. The $\tau$-jets with $\eta<3.0$ are then divided into several $E_{T}$ bins. A $\tau$-jet in any $E_{T}$ bin is then treated as tagged or untagged according to the efficiency $\left(\epsilon_{\tau}\right)$ given in [29] Fig. 12.9 for a particular bin.

We have implemented following selection criteria (see Chapter 13 of [4]):

- We select events with at least two jets having $\mathrm{P}_{\mathrm{T}}>150 \mathrm{GeV}\left(\mathrm{Cut} 1^{\prime}\right)$.

- Events with missing energy $\left(E_{T}\right)>200 \mathrm{GeV}$ are selected (Cut 2').

- Events with $M_{\text {eff }}>1000 \mathrm{GeV}$ are selected, where $M_{\text {eff }}=\left|\mathscr{H}_{T}\right|+\Sigma_{i}\left|P_{T}^{l_{i}}\right|+\Sigma_{i}\left|P_{T}^{j_{i}}\right|$ $(l=e, \mu)\left(\right.$ Cut $\left.3^{\prime}\right)$.

- Only events with jets having $\mathrm{S}_{\mathrm{T}}>0.2$, where $\mathrm{S}_{\mathrm{T}}$ is a standard function of the eigenvalues of the transverse sphericity tensor, are accepted (Cut $\left.4^{\prime}\right)$. 
For $m_{H^{ \pm}}=300 \mathrm{GeV}$ the $\mathrm{LO}$ production cross section is $1.29 \mathrm{pb}$. In Table 8 we present the effective cross sections $\left(\sigma \times \epsilon_{4}\right)$ of $0 l, 1 l$ and $1 \tau+X$ events after implementing the Cuts $\left(1^{\prime}\right.$ - $4^{\prime}$ ) for $m_{H^{ \pm}}=300 \mathrm{GeV}$. Here $H^{ \pm}$is allowed to decay in all possible modes. We find that the number of Higgs induced events for $\mathcal{L}_{\text {int }}=10 \mathrm{fb}^{-1}$ are 20.3, 7.5 and 6.9 respectively. These numbers though numerically significant, are unlikely to affect the result of SUSY search. Our earlier analyses clearly suggest that the strong $M_{\text {eff }}\left(\mathrm{Cut} 3^{\prime}\right)$ protects the squark-gluino signals from contamination due to Higgs induced events. The number of events for $0 l$ for $m_{H^{ \pm}}=400,500 \mathrm{GeV}$ at $\mathcal{L}_{\text {int }}=10 \mathrm{fb}^{-1}$ are 22 and 18 respectively.

\section{Conclusions}

In many models with extended Higgs sectors there are varities of new particles. It is then pertinent to ask how many events stemming purely from new particle production, can pass the slection criteria for Higgs search at the LHC, usually designed to remove the SM background alone. Such events ("the new physics" background) if sizable in numbers will obviously obfuscate the Higgs signal. Additional kinematic selection must, therefore, be carefully designed to suppress the new backgrounds while the bulk of the Higgs signal is retained.

It should also be stressed that before these additional cuts the Higgs and the new physics events together may stand above the SM background and reveal BSM physics at early stages of the LHC experiment, using selection criteria quite different from the cannonical search strategies for new physics.

We illustrate this very generic possibility by charged Higgs $\left(H^{ \pm}\right)$search in the MSSM taking into account the SUSY backgrounds from squark gluino events. In the decoupling regime $\left(m_{A} \gg m_{Z}\right)$ the lighter scalar $(h)$ mimics the SM Higgs boson. The first step for establishing the extented Higgs sector of the MSSM would, therefore, be to discover another Higgs boson. The early discovery of the charged Higgs can adequately serve this purpose. The larger the $m_{H}^{ \pm}$, the more challenging the discovery would be. Our main analysis is focussed on charged higgs search in the deep decoupling regime with $500 \leq m_{H^{ \pm}} \leq 800$ $\mathrm{GeV})$. We also comment on the prospect of lighter charged Higgs search in the presence of SUSY backgrounds.

There already exists in the literature several strategies to tame the SM background to the Higgs signal $[3,4,21]$. A variety of points in the MSSM parameter space with different 
characteristics including a mSUGRA point consistent with the observed DM relic density are considered (see Section 2) for sampling the SUSY backgrounds. The background events have a large number of taggable $\tau$-jets, b-jets and either genuine or fake reconstructed top quarks.

At the first stage of our analysis, we implement Cuts 1-7 (see Section 3, Table 3) following [21]. They suppress the SM backgrounds quite a bit, but- as is well known- are not enough to establish a statistically significant Higgs signal without additional cuts. If SUSY backgrounds are also present, the Higgs signal will be swamped by the combined background ( see $\sigma \times \epsilon_{1}$ in Tables 3, 4, 6 and 7). On the other hand the combined Higgs and squark-gluino events in the scenarios considered by us would stand above the SM background and establish BSM physics using selection criteria quite different from that typically implemented for SUSY search alone. In fact the outcome of this analysis would also disfavour the two Higgs doublet extension of the SM with comparable charged Higgs mass and BRs. Yet the new physics events will be an admixture of Higgs and squark-gluino events with no clear evidence of the Higgs signal.

In the standard analyses ignoring the SUSY background, the second stage consists of additional cuts which further suppress the SM background and establish the Higgs signal at a higher level of confidence. The polarization properties of the $\tau$ 's stemming from the charged Higgs decay are utilized in many analyses (see [23] and references there in). However, as argued in Section 3, the selections based on $\tau$-polarization may not be very efficient if SUSY backgrounds are present.

In lieu of $\tau$-polarization we select a more generic set of cuts (Cut 8-9, see Table 3) which depends on kinematics rather than on the compositions of the sparticles. In the absence of SUSY backgrounds, the estimated reach for $m_{H^{ \pm}}$on the basis of this generator level analysis would be close to $800 \mathrm{GeV}$ for $\mathcal{L}_{\text {int }}=30 \mathrm{fb}^{-1}$. A full simulation based on this alternative strategy would be welcome.

The above cuts not only bring the SM backgrounds further down but also enrich the fraction of the Higgs induced events in the surviving sample ( see $\sigma \times \epsilon_{2}$ in Tables 3, 4, 6 and 7). But the SUSY backgrounds may still be too large for an unambiguous Higgs discovery as is illustrated by the above examples. In fact even if the charged Higgs signal is too low, the SUSY background can fake it by standing above the SM background.

For selectively suppressing the SUSY events one has several alternatives. In Tables 3, 4, 
6 and 7 we display the effect of a cut on jet multiplicity (Cut 10) based on the fact that this number in a typical SUSY event is generally larger than that in the Higgs signal. After this cut an almost pure Higgs sample is left behind ( see $\sigma \times \epsilon_{3}$ in Tables 3, 4, 6 and 7).

In contrast if the size of the event sample remain practically unaltered after Cut 10, it would imply that no significant SUSY background was left behind after Cut 9. This by itself may be indicative of the nature of the SUSY parameter space. For example, scenarios with electroweak gauginos decaying via two body modes (see Section 2) into final states with energetic $\tau$-jets will be disfavoured.

The significance of the Higgs signal with respect to the scenario with the largest SUSY background (SUSY I )is displayed in Table 5. For $600 \leq m_{H^{ \pm}} \leq 800 \mathrm{GeV}$, the significance is $\geq 5$ for $\mathcal{L}_{i n t}=30 \mathrm{fb}^{-1}$. For $m_{H^{ \pm}}=500 \mathrm{GeV}, \mathcal{L}_{i n t} \sim 50 \mathrm{fb}^{-1}$ may be required.

We have also discussed several alternatives for finally suppressing the SUSY background. (see Table 5). The corresponding significance ( or the number of signal events if the background is zero) are also displayed in Table 5. It seems that the efficiency of the cut on jet multiplicity $\left(N_{\text {jet }} \leq 6\right)$ is marginally better. However, some selection criterion makes the signal background free according to our generator level calculation. If this conclusion survives a full simulation, then NLO cross sections would predict a signal size larger by a factor of 1.5 and the Higgs discovery could be made at a higher level of confidence.

It follows from Table 5 that the above strategy does not look very promising for lighter Higgs bosons $\left(m_{H^{ \pm}} \leq 500 \mathrm{GeV}\right)$. The main point is that for lower Higgs mass the $\tau$-jets are not sufficiently hard to pass the stiff $E_{T}$ cut (Cut 8). In such cases - after the standard cuts - one may implement the $\tau$-polarization cut which is indeed effective in removing the SM backgrounds. Finally Cut 10 or some of its alternatives may be used to further suppress the SUSY backgrounds to give a pure Higgs sample.

In conclusion we reiterate that the interplay between the Higgs and squark- gluino events could be relevant in principle for all Higgs (charged or neutral) search channels. More care in designing the selection procedure- keeping in mind the possible interplay between Higgs and squark-gluino events - for all the Higgs search channels is, therefore, called for. Since the key issue of electroweak symmetry breaking hinges on the Higgs sector this additional attention is indeed justified.

Acknowledgment: NB would like to thank the Council of Scientific and Industrial Research, Govt. of India for financial support. MM acknowledges support from Department 
of Science and Technology, India (grant SR/MF/PS-03/2009-VB-I).

\section{References}

[1] For review see, e.g. Perspectives in Supersymmetry, ed. G. L. Kane, World scientific (1998); Theory and Phenomenology of sparticles, M. Drees, R. M. Godbole and P. Roy, World Scientific (2004); Weak scale Supersymmetry: From superfields to scattering events, H. Baer and X. Tata, Cambridge UK, Univ. Press(2006).

[2] A. Djouadi, Phys. Rept. 459, 1 (2008); For a recent review and further references see, e.g., A. Djouadi and R. M. Godbole in Physics at the Large Hadron Collider, Indian Nataional Science Academy, A Platinum Jubilee Special Issue (Eds. A. Datta, B. Mukhopadhyaya and A. Raychaudhuri; Springer, 2009), p-47.

[3] For a review see, e.g., Higgs working group report (Les Houches 2003), K. A. Assamgon et al (hep-ph/0406152).

[4] A. De Roeck et al, CMS physics Technical Design Report, Vol-II, J. Phys. G 34, 995 (2007), Chapter 11.

[5] T. Sjostrand, P. Eden, C. Friberg, L. Lonnblad, G. Miu, S. Mrenna and E. Norrbin, Comp. Phys. Comm. 135, 238 (2001), For a more recent version see, (hep-ph/0603175).

[6] D. P. Roy, Phys. Lett. B 277, 183 (1992); Mod. Phys. Lett. A 19, 1813 (2004).

[7] A. H. Chamseddine, R. Arnowitt and P. Nath, Phys. Rev. Lett. 49, 970 (1982); R. Barbieri, S. Ferrara and C. A. Savoy, Phys. Rev. Lett. 119, 343 (1982); L. J. Hall, J. Lykken and S. Weinberg, Phys. Rev. D 27, 2359 (1983); P. Nath, R. Arnowitt and A. H. Chamseddine, Nucl. Phys. B 227, 121 (1983); N. Ohta, Prog. Theor. Phys. 70, 542 (1983).

[8] D. N. Spergel et al., Astrophys. J. Suppl. 170, 377 (2007).

[9] A. Djouadi, J. L. Kneur and G. Moultaka, Comput. Phys. Commun. 176, 426 (2007), [arXiv:hep-ph/0211331]. 
[10] J. Ellis and D. V. Nanopoulos, Phys. Lett. B 110, 44 (1982); F. Gabbiani, E. Gabriello, A. Masiero and L. Silvestrini, Nucl. Phys. B 477, 321 (1996); J. S. Hagelin et al, Nucl. Phys. B 415, 293 (1994); R. Barbieri, L. Hall, A. Strumia, Nucl. Phys. B 449, 437 (1995); M. Misiak, S. Pokorsky and J. Rosiek, Adv. Ser. Direct. High Energy Phys. 15, 795 (1998).

[11] J. M. Frere, D. R. T. Jones and S. Raby, Nucl. Phys. B 222, 11 (1983); J. A. Casas, A. Lleyda and C. Munoz, Nucl. Phys. B 471, 3 (1996).

[12] G. Belanger, F. Boudjema, A. Pukhov and A. Semenov, Comput. Phys. Commun. 176, 367 (2007) [arXiv:hep-ph/0607059].

[13] M. Drees and M. Nojiri, Phys. Rev. D 47, 376 (1993).

[14] Some comprehensive review articles are G. Jungman, M. Kamionkowski and K.Greist, Phys. Rep. 267, 195 (1996); L. Roszkowski, Pramana 62, 389 (2004); Manuel Drees, Plenary talk at 11th International Symposium on Particles, Strings and Cosmology (PASCOS 2005), Gyeongju, Korea, 30 May - 4 June 2005 (published in AIP Conf.Proc., 805, 48-54 (2006)(hep-ph/0509105); H. Baer and X. Tata in "Physics at the Large Hadron Collider", Indian Nataional Science Academy, A Platinum Jubilee Special Issue (Eds. A. Datta, B. Mukhopadhyaya and A. Raychaudhuri; Springer, 2009), p-179.

[15] J. R. Ellis, T. Falk, K. A. Olive and M. Srednicki, Astropart. Phys. 13, 181 (2000) [Erratum-ibid. 15, 413 (2001)]; R. Arnowitt, B. Dutta and Y. Santoso, Nucl. Phys. B 606, 59 (2001); T. Nihei, L. Roszkowski and R. Ruiz de Austri, J. High Energy Phys. 0207, 024 (2002).

[16] S. H. Zhu, Phys. Rev. D 67, 075006 (2003); T. Plehn, Phys. Rev. D 67, 014018 (2003); J. Alwall and J. Rathsman, J. High Energy Phys. 0412, 050 (2004), E. L. Berger, T. Han, J. Jiang and T. Plehn, Phys. Rev. D 71, 115012 (2005).

[17] H. L. Lai et al. [CTEQ Collaboration], Eur. Phys. J. C 12, 375 (2000) [arXiv:hepph/9903282], P.M. Nadolsky etal [CTEQ Collaboration], Phys. Rev. D 78, 013004 (2008). See also, http://www.phys.psu.edu/cteq.

[18] See, e.g. , A.Pukhov, CalcHEP-a package for evaluation of Feynman diagrams and integration over multi-particle phase space (hep-ph/9908288). 
For the more recent versions see: http://www.ifh.de/pukhov/calchep.html.

[19] M. Mangano et al. , J. High Energy Phys. 0307, 001 (003).

[20] see [4]; see also M. Baarmand et al. , CMS Note 2006/100.

[21] M. Guchait, R. Kinnunen and D. P. Roy, Eur. Phys. J. C 52, 665 (2007).

[22] B. K. Bullock, K. Hagiwara and A. D. Martin, Phys. Rev. Lett. 67, 3055 (1991); Nucl. Phys. B 395, 499 (1993).

[23] For a review see, e.g., M. Guchait and D. P. Roy in "Physics at the Large Hadron Collider", Indian Nataional Science Academy, A Platinum Jubilee Special Issue (Eds. A. Datta, B. Mukhopadhyaya and A. Raychaudhuri; Springer, 2009), p-205.

[24] S. Raychaudhuri, D. P. Roy, Phys. Rev. D 52, 1556 (1995); Phys. Rev. D 53, 4902 (1996); D. P. Roy, Phys. Lett. B 349, 607 (1999).

[25] M. M. Nojiri, Phys. Rev. D 51, 6281 (1995).

[26] M. Guchait and D. P. Roy, Phys. Rev. D 54, 6756 (1996); Phys. Lett. B 541, 356 (2002); R. M. Godbole, M. Guchait and D. P. Roy, Phys. Rev. D 79, 095015 (2009).

[27] M. Cacciari et al. , J. High Energy Phys. 404, 68 (2004); N.Kidonakis and R.Vogt, Phys. Rev. D 68, 114014 (2003).

[28] W. Beenakker, R. Hopker, M. Spira and P. M. Zerwas, Nucl. Phys. B 492, 51 ( 1997).

[29] D. Acosta, CMS Physics Technical Design Report, Vol-I, 2006. 